\title{
Existence theorems of an extension for generalized strong vector quasi-equilibrium problems
}

Kanokwan Sitthithakerngkiet ${ }^{1}$ and Somyot Plubtieng ${ }^{2^{*}}$

"Correspondence:

Somyotp@nu.ac.th

${ }^{2}$ Department of Mathematics,

Faculty of Science, Naresuan

University, Phitsanulok, 65000,

Thailand

Full list of author information is

available at the end of the article

\begin{abstract}
In this paper, we study generalized strong vector quasi-equilibrium problems in topological vector spaces. Using the generalization of Fan-Browder fixed point theorem, we provide existence theorems for an extension of generalized strong vector quasi-equilibrium problems with and without monotonicity. The results in this paper generalize, extend and unify some well-known existence theorems in literature. MSC: 49J30; 49J40; 47H10; 47H17
\end{abstract}

Keywords: quasi-equilibrium problems; variational inequality; monotonicity; Brouwer fixed point theorem

\section{Introduction}

The minimax inequalities of Fan [1] are fundamental in proving many existence theorems in nonlinear analysis. Their equivalence to the equilibrium problems was introduced by Takahashi [2, Lemma 1] Blum and Oettli [3] and Noor and Oettli [4]. The equilibrium problem theory provides a novel and united treatment of a wide class of problems which arise in economics, finance, image reconstruction, ecology, transportation, network, elasticity and optimization. This theory has had a great impact and influence in the development of several branches of pure and applied sciences. During this period, many results on existence of solutions for vector variational inequalities and vector equilibrium problems have been established (see, for example, [5-12]).

Recently, the equilibrium problem has been extensively generalized to the vector mappings (see $[6-8,10-16]$ ). Let $X$ and $Y$ be real topological vector spaces and $K$ be a nonempty subset of $X$. Let $C$ be a closed and convex cone in $Y$ with int $C \neq \emptyset$, where int $C$ denotes the topological interior of $C$. For a vector-value function $F: K \times K \rightarrow Y$, at least two different vector equilibrium problems are the following problems:

WVEP: find $x \in K$ such that $F(x, y) \notin-\operatorname{int} C$ for all $y \in K$

and

$V E P$ : find $x \in K$ such that $F(x, y) \notin-C \backslash\{0\} \quad$ for all $y \in K$.

The first problem is called weak vector equilibrium problem (see, for instance, $[13,17$, 18]) and the second one is normally called strong vector equilibrium problem (see [19]).

\section{照 Springer}

○2013Sitthithakerngkiet and Plubtieng; licensee Springer. This is an Open Access article distributed under the terms of the Creative Commons Attribution License (http://creativecommons.org/licenses/by/2.0), which permits unrestricted use, distribution, and reproduction in any medium, provided the original work is properly cited. 
However, Kazmi and Khan [20] called problem (1.2) the generalized system (for short, GS). Recently, many existence results extended and improved WVEP and its particular cases (see, for instance, [21-25]), but not VEP.

For a more general form of vector equilibrium problem, we let $A: K \rightarrow 2^{K}$ be a multivalued map with nonempty values where $2^{K}$ denotes the family of subsets of $K$. Then we consider the following problem: find $x \in K$ such that

$$
x \in A(x), \quad F(x, y) \notin-\operatorname{int} C \quad \text { for all } y \in A(x) .
$$

It is known that a vector quasi-equilibrium problem (for short, $V Q E P$ ) was introduced by Ansari et al. [23]. If the mapping $F$ is replaced by a multivalued map, saying $F: K \times K \rightarrow 2^{Y}$ with $Y$ being a topological vector space, $V E P$ can be generalized in the following way: find $x \in K$ such that

$$
F(x, y) \nsubseteq \nsubseteq-\operatorname{int} C \quad \text { for all } y \in K \text {. }
$$

It is called generalized vector equilibrium problem (for short, GVEP) and it has been studied by many authors; see, for example, $[14,15,21,22,26]$ and references therein. In 2003, Ansari and Flores-Bazás [21] introduced the generalized vector quasi-equilibrium problem (for short, GVQEP): find $x \in K$ such that

$$
x \in A(x), \quad F(x, y) \nsubseteq-\operatorname{int} C \quad \text { for all } y \in A(x),
$$

which is a general form of GVEP; more examples can also be found in [13-15, 26]. In another way, Kum and Wong [27] considered the multivalued generalized system (for short, $M G S)$ : find $x \in K$ such that

$$
F(x, y) \nsubseteq-C \backslash\{0\} \quad \text { for all } y \in K \text {. }
$$

Throughout this paper, unless otherwise specified, we assume that $X$ and $Y$ are Hausdorff topological vector spaces, $K$ is a nonempty convex subset of $X$ and $C$ is a pointed closed convex cone in $Y$ with int $C \neq \emptyset$. For a given multivalued bi-operator $F: K \times K \rightarrow 2^{Y}$ such that $\{0\} \subseteq F(x, x)$ for each $x \in K$, where $2^{Y}$ denotes the family of subsets of $Y$, the new type of generalized strong vector quasi-equilibrium problem (for short, GSVQEP) is the problem to find $x \in K$ such that

$$
x \in A(x), \quad F(x, y) \not \subset-C \backslash\{0\} \quad \text { for all } y \in A(x),
$$

where $A: K \rightarrow 2^{K}$ is a multivalued map with nonempty values. If we set $F(x, y)=\langle T x, \eta(y-$ $x)\rangle$ for all $x, y \in K$, then the GSVQEP reduces to the following generalized quasi-variational like inequality problem (for short, GQVLIP): find $x \in K$ such that

$$
x \in A(x), \quad\langle T x, \eta(y-x)| \nsubseteq-C \backslash\{0\} \quad \text { for all } y \in A(x),
$$

where $T: K \rightarrow 2^{L(X, Y)}$ is a multivalued mapping, $\eta: K \times K \rightarrow X$ is a nonlinear mapping and $L(X, Y)$ is denoted by the space of all continuous linear operators for $X$ to $Y$. This 
above formulation is the generalization of vector variational inequalities, variational-like inequality problems and vector complementarity problems in infinite dimensional spaces studied by many authors (see [28-30] and references therein).

The main motivation of this paper is to establish some existence results for a solution to the new type of the generalized strong vector quasi-equilibrium problems GSVQEP with and without monotonicity by using the generalization of Fan-Browder fixed point theorem.

\section{Preliminaries}

Let us recall some definitions and lemmas that are needed in the main results of this paper.

Definition 2.1 [31] Let $X$ and $Y$ be two topological vector spaces, and let $T: X \rightarrow 2^{Y}$ be a set-valued mapping.

(i) $T$ is said to be upper semicontinuous at $x \in X$ if for each $x \in X$ and each open set $V$ in $Y$ with $T(x) \subset V$, there exists an open neighborhood $U$ of $x$ in $X$ such that $T(y) \subset V$ for each $y \in U$.

(ii) $T$ is said to be lower semicontinuous at $x \in X$ if for each $x \in X$ and each open set $V$ in $Y$ with $T(x) \cap V \neq \emptyset$, there exits an open neighborhood $U$ of $x$ in $X$ such that $T(y) \cap V \neq \emptyset$ for each $y \in U$.

(iii) $T$ is said to be continuous on $X$ if it is at the same time upper semicontinuous and lower semicontinuous on $X$. It is also known that $T: X \rightarrow 2^{Y}$ is lower semicontinuous if and only if for each closed set $V$ in $Y$, the set $\{x \in X \mid T(x) \subset V\}$ is closed in $X$.

(iv) $T$ is said to be closed if the graph of $T$, i.e., $\operatorname{Graph}(T)=\{(x, y): x \in X$ and $y \in T(x)\}$, is a closed set in $X \times Y$.

Definition 2.2 [27] Let $X, Y$ be Hausdorff topological vector spaces, $K$ be a nonempty convex subset of $X$ and $C$ be a pointed closed convex cone in $Y$ with int $C \neq \emptyset$.

(i) A multivalued bi-operator $F: K \times K \rightarrow 2^{Y}$ is said to be $C$-strongly pseudomonotone if it satisfies

$$
\forall x, y \in K, \quad F(x, y) \nsubseteq \subseteq-C \backslash\{0\} \quad \Rightarrow \quad F(y, x) \subseteq-C .
$$

(ii) A multivalued mapping $G: K \rightarrow 2^{Y}$ is said to be $C$-convex if for all $x, y \in K$ and for all $\lambda \in[0,1]$,

$$
G(\lambda x+(1-\lambda) y) \subseteq \lambda G(x)+(1-\lambda) G(y)-C .
$$

And the mapping $G$ is said to be generalized hemicontinuous (in short, g.h.c.) if for all $x, y \in K$ and for all $\lambda \in[0,1]$,

$$
\lambda \mapsto G(x+\lambda(y-x)) \text { is upper semicontinuous at } 0^{+} .
$$

Definition 2.3 [27] Let $T: K \rightarrow 2^{L(X, Y)}$ and $\eta: K \times K \rightarrow X$ be nonlinear mappings. Then:

(i) $T$ is said to be hemicontinuous if, for any given $x, y, z \in K$ and for $\lambda \in[0,1]$, the mapping $\lambda \rightarrow\langle T(x+\lambda(y-z), z)\rangle$ is continuous at $0^{+}$; 
(ii) $T$ is said to be $C-\eta$-strongly pseudomonotone if, for any $x, y \in K$,

$$
\langle T x, \eta(y, x)| \nsubseteq-C \backslash\{0\} \quad \text { implies }\langle T y, \eta(x, y)\rangle \subseteq-C ;
$$

(iii) $\eta$ is said to be affine in the second argument if, for any $x_{i} \in K$ and $\lambda_{i} \geq 0(1 \leq i \leq n)$, with $\sum_{i=1}^{n} \lambda_{i}=1$ and any $y \in K, \eta\left(y, \sum_{i=1}^{n} \lambda_{i} x_{i}\right)=\sum_{i=1}^{n} \lambda_{i} \eta\left(y, x_{i}\right)$.

The following lemma is useful in what follows and can be found in [32].

Lemma 2.4 Let $X$ be a topological space and $Y$ be a set. Let $T: X \rightarrow 2^{Y}$ be a map with nonempty values. Then the following are equivalent:

(i) $T$ has the local intersection property;

(ii) There exits a map $F: X \rightarrow 2^{Y}$ such that $F(x) \subset T(x)$ for each $x \in X, F^{-1}(y)$ is open for each $y \in Y$ and $X=\bigcup_{y \in Y} F^{-1}(y)$.

Subsequently, Browder [33] obtained in 1986 the following fixed point theorem.

Theorem 2.5 (Fan-Browder fixed point theorem) Let $X$ be a nonempty compact convex subset of a Hausdorff topological vector space and $T: X \rightarrow 2^{X}$ be a map with nonempty convex values and open fibers (i.e., for $y \in Y, T^{-1}(y)$ is called the fiber of $T$ on $y$ ). Then $T$ has a fixed point.

The generalization of the Fan-Browder fixed point theorem was obtained by Balaj and Muresan [34] in 2005 as follows.

Theorem 2.6 Let $X$ be a compact convex subset of a topological vector space and $T: X \rightarrow$ $2^{X}$ be a map with nonempty convex values having the local intersection property. Then $T$ has a fixed point.

Lemma 2.7 [35] Let $X$ be a bounded subset of E. Then the usual pairing $\langle\cdot, \cdot\rangle: E^{*} \times X \rightarrow R$ is continuous.

\section{Main theorem}

In this section, we shall investigate the existence results for GSVQEP and GQVLIP with monotonicity and without monotonicity. First, we present the following lemma which is of Minty's type for GSVQEP.

Lemma 3.1 Let $K$ be a nonempty and convex subset of $X$, let $A: K \rightarrow 2^{K}$ be a set-valued mapping such that for any $x \in K, A(x)$ is a nonempty convex subset of $K$ and let $F: K \times$ $K \rightarrow 2^{y}$ be g.h.c. in the first argument, $C$-convex in the second argument and C-strongly pseudomonotone. Then the following problems are equivalent:

(i) Find $x \in K$ such that $x \in A(x), F(x, y) \nsubseteq \subseteq-C \backslash\{0\}, \forall y \in A(x)$.

(ii) Find $x \in K$ such that $x \in A(x), F(y, x) \subseteq-C, \forall y \in A(x)$.

Proof (i) $\rightarrow$ (ii) It is clear by the $C$-strong pseudomonotonicity.

(ii) $\rightarrow$ (i) Let $x \in K$. For any $y \in A(x)$ and $\theta \in(0,1)$, we set $z_{\theta}=\theta y+(1-\theta) X$. By the assumption (ii) and the convexity of $A(x)$, we conclude that

$$
x \in A(x), \quad F\left(z_{\theta}, x\right) \subseteq-C .
$$


Since $F$ is $C$-convex in the second argument, we have

$$
\begin{aligned}
0 & \in F\left(z_{\theta}, z_{\theta}\right) \\
& \subseteq \theta F\left(z_{\theta}, y\right)+(1-\theta) F\left(z_{\theta}, x\right)-C \\
& \subseteq \theta F\left(z_{\theta}, y\right)-C .
\end{aligned}
$$

Then we have $F\left(z_{\theta}, y\right) \cap C \neq \emptyset$, because $C$ is a convex cone. Since $F$ is g.h.c. in the first argument, we have $x \in A(x), F(x, y) \cap C \neq \emptyset, \forall y \in A(x)$. It implies that $x \in A(x), F(x, y) \nsubseteq$ $-C \backslash\{0\}$ for all $y \in A(x)$. This completes the proof.

In the following theorem, we present the existence result for GSVQEP by assuming the monotonicity of the function.

Theorem 3.2 Let $K$ be a nonempty compact convex subset of $X$. Let $A: K \rightarrow 2^{K}$ be a setvalued mapping such that for any $x \in K, A(x)$ is a nonempty convex subset of $K$ and for each $y \in K, A^{-1}(y)$ is open in $K$. Let the set $P:=\{x \in X \mid x \in A(x)\}$ be closed. Assume that $F: K \times K \rightarrow 2^{Y}$ is $C$-strongly pseudomonotone, g.h.c. in the first argument, $C$-convex and l.s.c. in the second argument. Then GSVQEP has a solution.

Proof For any $x \in K$, we define the set-valued mapping $S, T: K \rightarrow 2^{K}$ by

$$
\begin{aligned}
& S(x)=\{y \in K \mid F(y, x) \nsubseteq \subseteq-C\}, \\
& T(x)=\{y \in K \mid F(x, y) \subseteq-C \backslash\{0\}\},
\end{aligned}
$$

and for any $y \in K$, we denoted the complement of $S^{-1}(y)$ by $\left(S^{-1}(y)\right)^{C}=\{x \in K \mid F(y, x) \subseteq$ $-C$. For each $x \in K$, we define multivalued maps $G, H: K \rightarrow 2^{K}$ by

$$
G(x)= \begin{cases}S(x) \cap A(x) & \text { if } x \in P \\ A(x) & \text { if } x \in K \backslash P\end{cases}
$$

and

$$
H(x)= \begin{cases}T(x) \cap A(x) & \text { if } x \in P, \\ A(x) & \text { if } x \in K \backslash P .\end{cases}
$$

Clearly, $G(x)$ and $H(x)$ are nonempty sets for all $x \in K$, and by the $C$-strong pseudomonotonicity of $F$, we have $G(x) \subseteq H(x)$ for all $x \in K$. We claim that $H(x)$ is convex. Let $y_{1}, y_{2} \in T(x)$ and $\theta \in(0,1)$. Since $F$ is $C$-convex in the second argument, we have

$$
\begin{aligned}
F\left(x, \theta y_{1}+(1-\theta) y_{2}\right) & \subseteq \theta F\left(x, y_{1}\right)+(1-\theta) F\left(x, y_{2}\right)-C \\
& \subseteq(-C \backslash\{0\})-C \\
& \subseteq-C \backslash\{0\} .
\end{aligned}
$$

Then we have $T(x)$ is convex and so $H(x)$ is convex by the convexity of $A(x)$. Next, we will show that $G^{-1}(y)$ is open in $K$ for each $y \in K$. Since $F$ is l.s.c. in the second argument 
and by the definition of $\left(S^{-1}(y)\right)^{C}$, we have $\left(S^{-1}(y)\right)^{C}$ closed and so $S^{-1}(y)$ is open in $K$. By assumption, we obtain that

$$
G^{-1}(y)=\left(S^{-1}(y) \cap A^{-1}(y)\right) \cup\left(A^{-1}(y) \cap K \backslash P\right)
$$

is open in $K$. It is easy to see that the mapping $H$ has no fixed point because $0 \in F(x, x)$, $\forall x \in K$. From the contrapositive of the generalization of the Fan-Browder fixed point theorem and Lemma 2.4, we have

$$
K \nsubseteq \bigcup_{y \in K} G^{-1}(y)
$$

Hence, there exists $\bar{x} \in K$ such that $G(\bar{x})=\emptyset$. If $\bar{x} \in K \backslash P$, we have $A(\bar{x})=\emptyset$, which contradicts the assumptions. Then $\bar{x} \in P$ and hence $S(\bar{x}) \cap A(\bar{x})=\emptyset$. This means that $\bar{x} \in A(\bar{x})$ and $F(y, \bar{x}) \subseteq-C$ for all $y \in A(\bar{x})$. This completes the proof by Lemma 3.1.

The following example shows that GSVQEP has a solution under the condition of Theorem 3.2.

Example 3.3 Let $Y=\mathbb{R}, C=[0, \infty)$ and $K=[-1,1]$. Define the mapping $A: K \rightarrow 2^{K}$ and $F: K \times K \rightarrow 2^{Y}$ by

$$
A(x)= \begin{cases}{[-0.5, x+0.5)} & \text { if }-1 \leq x<0 \\ (-0.5,0.5) & \text { if } x=0, \\ (x-0.5,0.5] & \text { if } 0<x \leq 1\end{cases}
$$

and

$$
F(x, y)= \begin{cases}{[0, y-x]} & \text { if } x<y, \\ {[y-x, 0]} & \text { if } x \geq y,\end{cases}
$$

respectively. By the definition of $A$, see Figure 1 , we have the set $P=\{x \in X \mid x \in A(x)\}=$ $[-0.5,0.5]$ which is closed and for each $y \in K, A^{-1}(y)$ is open in $K$.

We see that $F$ is $C$-strongly pseudomonotone. Indeed, if $F(x, y) \nsubseteq \subseteq C \backslash\{0\}$, then we only consider the case $x<y$, so $F(x, y)=[0, y-x]$. That is,

$$
F(y, x)=[x-y, 0] \subseteq-C \text { for all } x<y .
$$

Figure 1 The image set $A(x)$ for all $x$ in $K$.

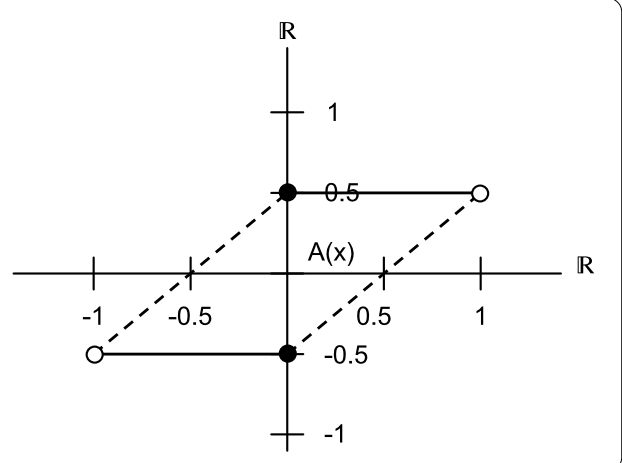


Let $x, y, z \in K$ and $\lambda \in[0,1]$. If $x<\lambda y+(1-\lambda) z$, then

$$
\begin{aligned}
F(x, \lambda y+(1-\lambda) z) & =[0, \lambda y+(1-\lambda) z-x] \\
& =[0, \lambda(y-x)+(1-\lambda)(z-x)] \\
& \subseteq[0, \lambda(y-x)+(1-\lambda)(z-x)]-C \\
& =\lambda[0, y-x]+(1-\lambda)[0, z-x]-C \\
& =\lambda F(x, y)+(1-\lambda) F(x, y)-C .
\end{aligned}
$$

Similarly, in another case, we have $F$ is C-convex in the second argument. Clearly, $F$ is g.h.c. in the first argument and l.s.c. in the second argument.

Moreover, this example asserts that -0.5 is one of the solutions because if $x=-0.5$, then $A(x)=[-0.5,0)$. Note that for all $y \in A(x), y>x$. Therefore $F(-0.5, y)-[0, y+0.5] \nsubseteq \subseteq-C \backslash\{0\}$ for all $y \in[-0.5,0)$.

Now, we present an existence theorem for GSVQEP when $F$ is not necessarily monotone.

Theorem 3.4 Let $K$ be a nonempty compact convex subset of $X$, let $A: K \rightarrow 2^{K}$ be a setvalued mapping such that for each $x \in K, A(x)$ is a nonempty convex subset of $K$, and let the set $P:=\{x \in X \mid x \in A(x)\}$ be closed. Assume that $F: K \times K \rightarrow 2^{Y}$ is $C$-convex in the second argument and for each $y \in K$, the set $\{x \in K \mid F(x, y) \subseteq-C \backslash\{0\}\}$ is open. Then GSVQEP has a solution.

Proof We proceed with the contrary statements, that is, for each $x \in X, x \notin A(x)$ or there exists $y \in A(x)$ such that

$$
F(x, y) \subseteq-C \backslash\{0\}
$$

For every $y \in K$, we define the sets $N_{y}$ and $M_{y}$ as follows:

$$
N_{y}:=\{x \in K: F(x, y) \subseteq-C \backslash\{0\}\}
$$

and

$$
M_{y}:=N_{y} \cup P^{C} .
$$

By the assumption, we have the set $M_{y}$ is open in $K$ and we see that $\left\{M_{y}\right\}_{y \in K}$ is an open cover of $K$. Since $K$ is compact, there exists a finite subcover $\left\{M_{y_{i}}\right\}_{i=1}^{n}$ such that $K=\bigcup_{i=1}^{n} M_{y_{i}}$. By a partition of unity, there exists a family $\left\{\beta_{i}\right\}_{i=1}^{n}$ of real-valued continuous functions subordinate to $\left\{M_{y_{i}}\right\}_{i=1}^{n}$ such that for all $x \in K, 0 \leq \beta_{i}(x) \leq 1$ and $\sum_{i=1}^{n} \beta_{i}(x)=1$ and for each $x \notin M_{y_{i}}, \beta_{i}(x)=0$. Let $C:=\operatorname{co}\left\{y_{1}, y_{2}, \ldots, y_{n}\right\} \subseteq K$. Then $C$ is a simplex of a finite dimensional space. Define a mapping $S: C \rightarrow C$ by

$$
S(x)=\sum_{i=1}^{n} \beta_{i}(x) y_{i}, \quad \forall x \in C .
$$


Hence, we have $S$ is continuous since $\beta_{i}$ is continuous for each $i$. From Brouwer's fixed point theorem, there exists $x_{0} \in C$ such that $x_{0}=S\left(x_{0}\right)$. We define a set-valued mapping $T: K \rightarrow 2^{Y}$ by

$$
T(x)=F(x, S(x)) \quad \text { for all } x \in K
$$

Now, we note that for any $x \in K,\left\{y_{i} \mid x \in M_{y_{i}}\right\} \neq \emptyset$. Since $F$ is $C$-convex in the second argument, it follows from (3.1), (3.2) and (3.3) that we have

$$
\begin{aligned}
T(x) & =F\left(x, \sum_{i=1}^{n} \beta_{i}(x) y_{i}\right) \\
& \subseteq \sum_{i=1}^{n} \beta_{i}(x) F\left(x, y_{i}\right)-C \\
& \subseteq-C \backslash\{0\}-C \\
& =-C \backslash\{0\}
\end{aligned}
$$

for all $x \in K$. Since $x_{0} \in K$ and it is a fixed point of $S, 0=F(x, x)=F(x, S(x))=T(x) \subseteq$ $-C \backslash\{0\}$, which is a contradiction. This completes the proof.

If we set $A \equiv I$, then Theorem 3.2 and Theorem 3.4 are reduced to Theorem 1 and Theorem 3 in Kum and Wong [27], respectively. Moreover, Theorem 3.2 is a multivalued version of Theorem 2.3 in Kazmi and Khan [20].

Let $F(x, y)=\langle T x, \eta(y, x)\rangle$ for all $x, y \in K$, where $\eta: K \times K \rightarrow X$ and $T: K \rightarrow 2^{L(X, Y)}$. As a consequence of Theorem 3.2 and using the same argument as in Kum and Wang ([27], Theorem 2), we have the following existence result for GQVLIP.

Corollary 3.5 Let $K$ be a nonempty compact convex subset of $X$, let $A: K \rightarrow 2^{K}$ be a setvalued mapping such that for any $x \in K, A(x)$ is a nonempty convex subset of $K$ and for each $y \in K, A^{-1}(y)$ is open in $K$. Let the set $P:=\{x \in X \mid x \in A(x)\}$ be closed, let $\eta: K \times K \rightarrow X$ be affine and continuous in the first argument and hemicontinuous in the second argument, and let $T: K \rightarrow 2^{L(X, Y)}$ be a C-strongly pseudomonotone and g.h.c. with nonempty compact values where $L(X, Y)$ is equipped with topology of bounded convergence. Then GQVLIP has a solution.

As a consequence of Theorem 3.4, we obtain the following existence result for GQVLIP.

Corollary 3.6 Let $K$ be a nonempty compact convex subset of $X$. Let $A: K \rightarrow 2^{K}$ be a setvalued mapping such that for each $x \in X, A(x)$ is a nonempty convex subset of $K$ and let the set $P:=\{x \in X \mid x \in A(x)\}$ be closed. Assume that $\eta: K \times K \rightarrow X$ is affine in the first argument and $T: K \rightarrow 2^{L(X, Y)}$ is a nonlinear mapping such that, for every $y \in K$, the set $\{x \in K \mid\langle T(x), \eta(y, x)\rangle \subseteq-C \backslash\{0\}\}$ is open. Then GQVLIP has a solution. 


\section{Authors' contributions}

The work presented here was carried out in collaboration between all authors. SP designed theorems and methods of the proof and interpreted the results. KS proved the theorems, interpreted the results and wrote the paper. All authors read and approved the final manuscript.

\section{Author details}

'Department of Mathematics, Faculty of Applied Science, King Mongkut's University of Technology North Bangkok, Bangkok, 10800, Thailand. '² Department of Mathematics, Faculty of Science, Naresuan University, Phitsanulok, 65000, Thailand.

\section{Acknowledgements}

The first author would like to thank the King Mongkut's University of Technology North Bangkok, Thailand.

\section{Received: 27 August 2013 Accepted: 21 November 2013 Published: 13 Dec 2013}

\section{References}

1. Fan, K: Minimax theorems. Proc. Natl. Acad. Sci. USA 39, 42-47 (1953)

2. Takahashi, W: Nonlinear variational inequalities and fixed point theorems. J. Math. Soc. Jpn. 28, 168-181 (1976)

3. Blum, E, Oettli, W: From optimization and variational inequalities to equilibrium problems. Math. Stud. 63, 123-145 (1994)

4. Noor, MA, Oettli, W: On general nonlinear complementarity problems and quasi-equilibria. Matematiche 49, 313-331 (1994)

5. Ansari, $\mathrm{OH}$, Yao, JC: On nondifferentiable and nonconvex vector optimization problems. J. Optim. Theory Appl. 106 487-500 (2000)

6. Fu, JY: Generalized vector quasi-equilibrium problems. Math. Methods Oper. Res. 52, 57-64 (2000)

7. Giannessi, F: Vector Variational Inequalities and Vector Equilibria. Mathematical Theories. Kluwer Academic, Dordrecht (2000)

8. Gong, XH: Strong vector equilibrium problems. J. Glob. Optim. 36, 339-349 (2006)

9. Lee, GM, Kim, DS, Kuk, H: Existence of solutions for vector optimization problems. J. Math. Anal. Appl. 220, 90-98 (1998)

10. Li, SJ, Teo, KL, Yang, XQ: Generalized vector quasi-equilibrium problem. Math. Methods Oper. Res. 61, $385-397$ (2005)

11. Long, XJ, Huang, NJ, Teo, KL: Existence and stability of solutions for generalized strong vector quasi-equilibrium problems. Math. Comput. Model. 47, 445-451 (2008)

12. Wan, $\mathrm{AH}, \mathrm{Fu}, \mathrm{JY}, \mathrm{Mao}, \mathrm{WH}$ : On generalized vector equilibrium problems. Acta Math. Appl. Sinica (Engl. Ser.) 22, 21-26 (2006)

13. Ansari, QH, Konnov, IV, Yao, JC: Characterizations of solutions for vector equilibrium problems. J. Optim. Theory Appl. 113, 435-447 (2002)

14. Ansari, QH, Schaible, S, Yao, JC: Generalized vector equilibrium problems under generalized pseudomonotonicity with applications. J. Nonlinear Convex Anal. 3, 331-344 (2002)

15. Konnov, IV, Yao, JC: Existence of solutions for generalized vector equilibrium problems. J. Fixed Point Theory Appl. 233, 213-221 (1999)

16. Song, W: On generalized vector equilibrium problems. J. Comput. Appl. Math. 146, 167-177 (2002)

17. Chen, GY, Hou, SH: Existence of solutions for vector variational inequalities. In: Giannessi, F (ed.) Vector Variational Inequalities and Vector Equilibria, pp. 73-86. Kluwer Academic, Dordrecht (2000)

18. Fang, YP, Huang, NJ: Strong vector variational inequalities in Banach spaces. Appl. Math. Lett. 19, $362-368$ (2006)

19. Bigi, G, Capata, A, Kassay, G: Existence results for strong vector equilibrium problems and their applications. Optimization 61(5), 567-583 (2012)

20. Kazmi, KR, Khan, SA: Existence of solution to a generalized system. J. Optim. Theory Appl. 142, 355-361 (2009)

21. Ansari, QH, Flores-Bazán, F: Generalized vector quasi-equilibrium problems with applications. J. Math. Anal. Appl. 277 246-256 (2003)

22. Ansari, QH, Konnov, IV, Yao, JC: On generalized vector equilibrium problems. Nonlinear Anal. 47, 543-554 (2001)

23. Ansari, QH, Yao, JC: On vector quasi-equilibrium problems. In: Maugeri, A, Giannessi, F (eds.) Variational Problems and Equilibria. Kluwer Academic, Dordrecht (2003)

24. Bianchi, M, Kassay, G, Pini, R: Ekeland's principle for vector equilibrium problems. Nonlinear Anal. 66, 1454-1464 (2007)

25. Tanaka, T: Existence results of cone saddle-points for vector-valued functions. Appl. Math. Optim. 36, 313-322 (1997)

26. Ansari, QH, Oettli, W, Schiager, D: A generalization of vector equilibrium. Math. Methods Oper. Res. 46, 147-152 (1997)

27. Kum, S, Wong, MM: An extension of a generalized equilibrium problem. Taiwan. J. Math. 15, 1667-1675 (2011)

28. Huang, NJ, Li, J: On vector implicit variational inequalities and complementarity problems. J. Glob. Optim. 34, 399-408 (2004)

29. Lee, GM, Kim, DS, Lee, BS: Generalized vector variational inequality. Appl. Math. Lett. 9, 39-42 (1996)

30. Yang, XQ: Vector variational inequality and its duality. Nonlinear Anal. 95, 729-734 (1993)

31. Berge, C: Topological Spaces. Oliver \& Boyd, Edinburgh (1963)

32. Ding, XP: A coincidence theorem involving contractible space. Appl. Math. Lett. 10, 53-56 (1997)

33. Browder, FE: The fixed point theory of multivalued mapping in topological vector spaces. Math. Ann. 177, 238-301 (1968)

34. Balaj, M, Muresan, S: Generalizations of the Fan-Browder fixed point theorem and minimax inequalities. Arch. Math. 41, 399-407 (2005)

35. Kum, S: A generalization of generalized quasi-variational inequalities. J. Math. Anal. Appl. 182, 158-164 (1994)

10.1186/1687-1812-2013-342

Cite this article as: Sitthithakerngkiet and Plubtieng: Existence theorems of an extension for generalized strong vector quasi-equilibrium problems. Fixed Point Theory and Applications 2013, 2013:342 